\title{
The Test and Training Enabling Architecture, TENA, Enabling Technology For The Joint Mission Environment Test Capability (JMETC) and Other Emerging Range Systems
}

\author{
By \\ Gene Hudgins \\ TENA Software Development Activity (SDA) \\ 557 Mary Esther Cut-Off \\ Ft Walton Beach FL 32548 \\ 850.244.7765 (Voice) 850.244.7590 (FAX)
}

\begin{abstract}
The Joint Mission Environment Test Capability (JMETC) is a distributed live, virtual, and constructive (LVC) testing capability developed to support the acquisition community and to demonstrate Net-Ready Key Performance Parameters (KPP) requirements in a customer-specific Joint Mission Environment (JME). JMETC, using the Test and Training Enabling Architecture, TENA, provides connectivity to the Services' distributed test capabilities and simulations, and industry test resources. TENA is well-designed for supporting JMETC events through its architecture and software capabilities which enable interoperability among range instrumentation systems, facilities, and simulations. TENA, used in major exercises and distributed test events, is also interfacing with other emerging range systems, such as iNET.
\end{abstract}

\section{KEY WORDS}

TENA, JMETC, interoperability, resource reuse, iNet

\section{INTRODUCTION}

The United States Department of Defense (DoD) has invested millions of dollars in test, training, and evaluation ranges. Geographically dispersed, spread from the Atlantic to the Pacific and from the Canadian border to the Mexican border and including Hawaii, Alaska, and the United States territories, the air, land, and sea ranges are used by the U.S. Military Services and various other agencies with training and equipment needs that must be resolved and validated. Today's warfighters test and train on these ranges and today's military test and training events range from individual systems under test to small-unit maneuvering to large-scale Joint Services exercises where simulated and constructive and live-fire events are blended to enact representative scenarios spread across several states. Critical data collected during these events provides weapon systems evaluation and validation, and perhaps more importantly, can quickly and definitively illuminate any necessary improvements to ensure effective and safe weapon system operation and training. This data also invariably affects almost every aspect of range operation and management, including budget definition and approval.

Being successful in the development of any Joint testing capability requires a supporting and guiding activity, and in December 2005, the JMETC program element was formed. JMETC, the DoD corporate approach for linking distributed facilities, is a distributed live, virtual, and constructive (LVC) testing capability developed to support the acquisition community during program development, developmental testing, operational testing, interoperability certification, 
and including demonstration of Net Ready Key Performance Parameters (KPP) requirements in a customer-specific Joint Mission Environment (JME). JMETC will provide readily available connectivity to the Services' distributed test capabilities and simulations, as well as industry test resources. JMETC, although a testing capability, is also aligned with and complemented by the Joint National Training Capability (JNTC) integration solutions to foster test, training, and experimental collaboration.

The JMETC program has used the Test and Training Enabling Architecture, TENA, to prototype new testing support infrastructure. TENA, the live range instrumentation architecture for JNTC and field-proven in major field exercises as well as numerous distributed test events, provides JMETC a technology already being deployed in DoD. TENA provides the middleware and software component while the JMETC Virtual Private Network (VPN) provides the hardware connectivity through utilization of the existing Secure Defense Research and Engineering Network (SDREN) and Defense Research and Engineering Network (DREN) infrastructure. As each VPN node is brought up, the JMETC team uses network testing tools: NUTTCP, Mping, and the JMETC Interface Verification Tool (IVT). NUTTCP, which requires a Unix operating system, is a TCP/UDP network performance tool, and Mping, version 2 is available from Microsoft and requires Windows XP, is used to test multicast capability between JMETC VPN sites. NUTTCP and Mping need to reside in the lab participating in the testing event. The IVT is used to test the VPN with TENA and/or other data protocols.

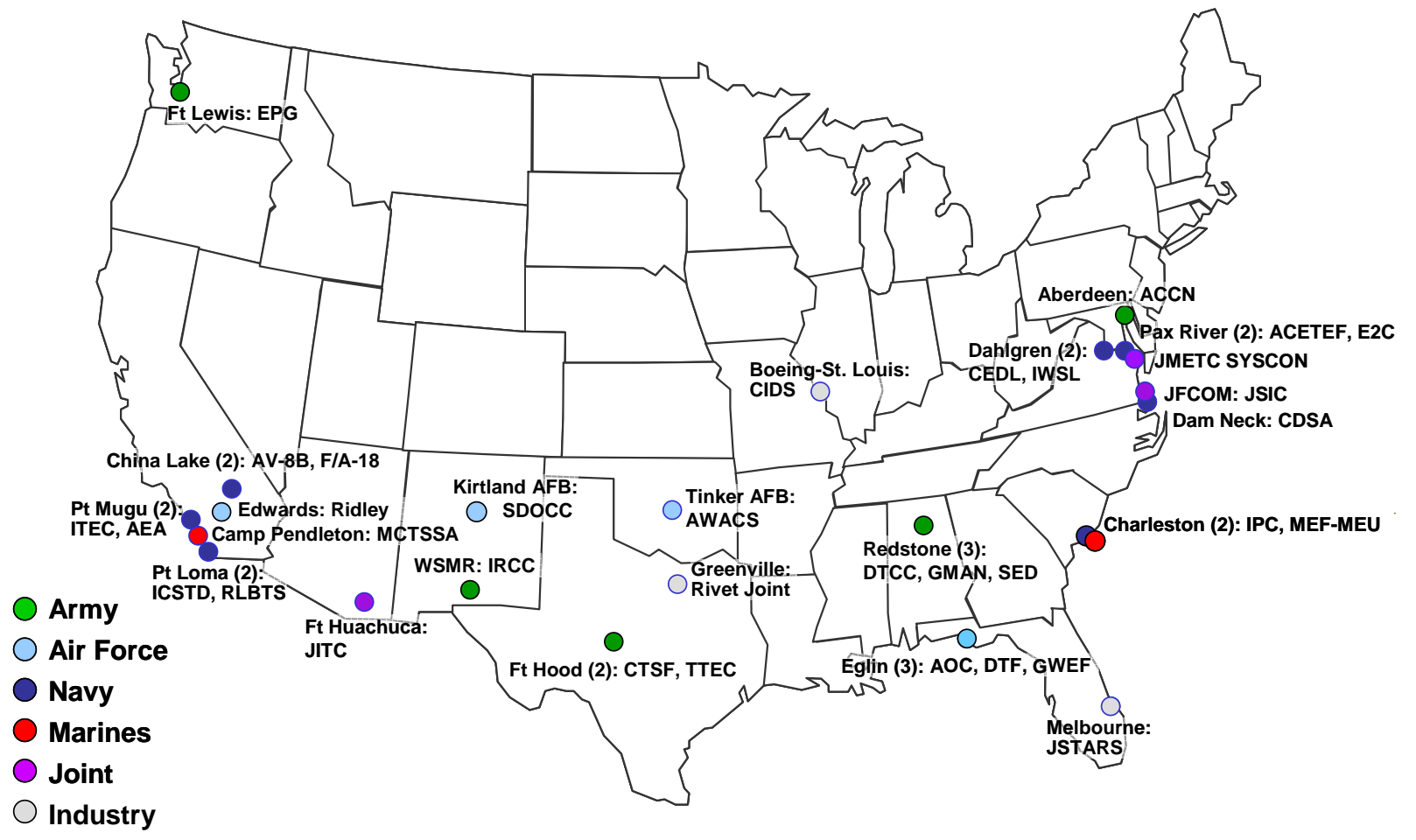

Figure 1. FY08 JMETC VPN 
JMETC VPN operational testing uses the IVT and TENA. Operational testing is performed by the User Support Team to verify the network can operationally support TENA and/or other data protocols. The testing is conducted after the network infrastructure test have been successfully performed by the JMETC network system control and will ensure the backbone JMETC VPN network (Service Delivery Point to Service Delivery Point) and the end-user site network infrastructures are configured for proper and efficient TENA operations. The operational testing is executed in two phases: Phase 1: one-on-one with each new or updated JMETC VPN site, Phase 2: full mesh with all sites to participate in a particular event/ exercise.

The JMETC Team's network goal is to complete a VPN infrastructure of 36 connected nodes during 2008. See Figure 1. Recently added sites include the Aberdeen Common Control Node; Kirkland AFB Air Force Operational Test and Evaluation Center; Boeing Center for Integrated Defense Simulation at St. Louis, MO; and two sites at Fort Hood, Texas. Based on the customer's needs and the potential for reuse, a dedicated and trusted VPN is provided on the SDREN, which is part of the Global Information Grid (GIG). The VPN sites, encrypted for Secret, also include numerous sites at Defense industrial facilities. This infrastructure can be connected to a Joint National Test Capability (JNTC)-sponsored Network Aggregator to further increase the VPN capability by bridging to sites on other classified networks to include JNTC Joint Test and Experimentation Network (JTEN), Defense Information System Network (DISN) networks, the Air Force Integrated Collaborative Environment (AF-ICE) enclave, and other classified enclaves.

Together, the TENA and JMETC complement enables and enhances distributed testing and training. While JMETC is a relatively new presence for the test and training community, TENA has evolved since the late 1990s when it was brought into play to solve an old problem that restricted range effectiveness. Many of the early range data collection and analysis systems were part of a vertical "stovepipe" growth of the instrumentation and instrumentation suites, and not able to utilize the advantages found in the concepts of range interoperability and range resource reuse, concepts that allow for taking easy advantage of the growth in modeling and simulation and its revolutionary application to training, concepts that were being forwarded in the late 1990s by the Foundation Initiative 2010 (FI 2010) project, which was sponsored by the Office of the Secretary of Defense (OSD) Central Test and Evaluation Investment Program (CTEIP).

Utilizing TENA, JMETC enabled several initial prototype demonstrations: an Air Combat example (a Data Link Messages Test Environment), Technical Alignment with JNTC events (test and training collaboration), a Land Combat example (Future Combat System (FCS) test environment), and an Information Operations example (IO Range integration). In August 2007, JMETC supported its “Stand Up” event, Integral Fire 07. Integral Fire 07 (IF07), an Air Force Integrated Collaborative Environment (AF-ICE) event, was a distributed test event involving all the military services and the U.S. Joint Forces Command (JFCOM). Recently, JMETC has supported the Joint Command, Control, Communications, Computers, Intelligence, Surveillance, and Reconnaissance (JC4ISR) Interoperability Test and Evaluation Capability (InterTEC) project Spiral 2, software build 2 System Integration Test (SIT-1) at Fort Huachuca, Arizona, and Point Mugu, California. InterTEC presents an integrated test solution for scaleable, extensible, and operationally relevant interoperability test and evaluation.

The earlier Air Combat example demonstrated distributed interoperability testing using a LVC environment. The Technical Alignment events with JNTC demonstrated collaboration on integration of tactical training range instrumentation for the Weapons \& Tactics Instructor (WTI) 
and Red Flag Alaska exercises. The Land Combat example, with JMETC support of the Army Cross-Command Collaborative Environment (3CE), demonstrated interoperable laboratories and ranges with common modeling and simulation and data environments supporting distributed LVC tests. The Information Operations (IO) Range Integration example, showed collaboration on Information Operations Use Cases and demonstrates network connectivity for large-scale, multiple security level events. TENA was used to control and distribute video data at multiple sites.

For the land Combat example, JMETC supported the 2006 US Army 3CE Simulation Environment Characterization Assessment (SECA) test event. The support to 3CE was provided for the following JMETC tools and utilities:

- Interface Verification Tool (IVT),

- $\quad$ TENA Middleware in a 3CE environment, and

- $\quad$ TENA-HLA gateway development using the Gateway Builder tool.

The SECA provided a means to integrate 3CE activities and provided a quantitative assessment of Standard Operating Procedures, the 3CE Capability Development/System Engineering Process, Analytic Data Requirement Process, Model Selection Process, and 3CE Interoperability. Some of the JMETC objectives of the event were to demonstrate that the DREN could support distributed LVC testing, demonstrate more efficient technical integration using JMETC Core infrastructure aspects, demonstrate TENA capability of supporting distributed LVC test activities, and demonstrate TENA interoperability with HLA-based simulations. Figure 2 illustrates the networked sites and systems used in 3CE SECA.

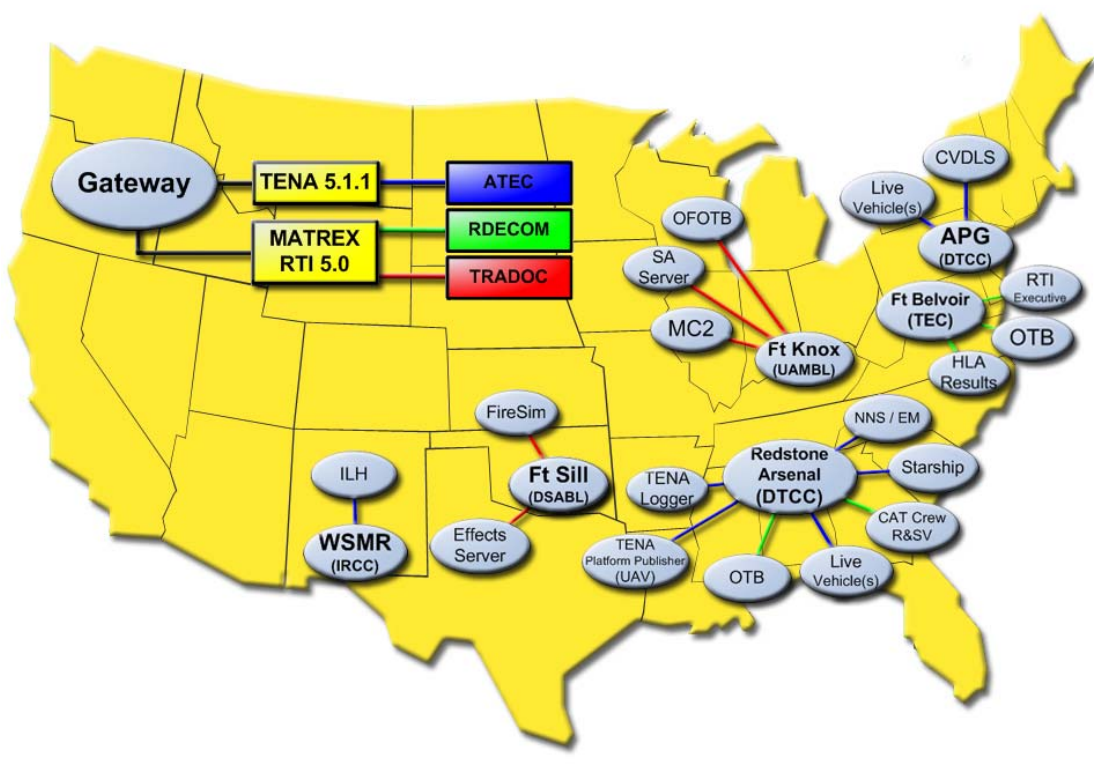

Figure 2. 3CE SECA Site Map

A JMETC technical team, including members of the TENA User Support Team, supported the integration of assets from three Army commands bridging Analysis, Research \& Development, and Testing \& Evaluation to integrate modeling and simulation across the acquisition process 
and provided common tools required for distributed testing. Based on the activities supporting the SECA integration, the following JMETC Objectives were verified:

- The DREN was proven stable, and once configured to support multi-cast, it has proven to be robust and reliable for distributed LVC testing.

- $\quad$ The JMETC Technical team demonstrated the value of JMETC-provided tools and utilities when executing pre-test integration activities, troubleshooting integration issues, and executing post-test procedures.

- Throughout the event planning and execution process, the TENA website repository provided excellent online collaboration for 3CE team members.

JMETC played an important enabling role in the Joint National Training Capability (JNTC) Austere Challenge 2006 (AC06) exercise. A TENA-enabled Video Distribution System (VDS) was used to provide a distributed visualization capability to the Information Operation (IO) Range in the event. Development of the IO Range VDS was accomplished through collaboration between the IO Range and the JMETC programs, resulting in a first time achievement of bringing real-time visualization of live fire IO events to the combatant commanders (COCOM), both CONUS and OCONUS, via the Secret Internet Protocol Router Net (SPIRNet).

The IO Range Video Distribution System (VDS) concept was based on an early TENA Data Stream Framework prototyped in FY2004 and leveraged a non-TENA Video Capture \& Distribution System being developed for the Redstone Technical Test Center (RTTC). The JMETC development team was able to quickly adapt their solution using TENA and required less than four weeks to develop, implement, test, and integrate for the exercise.

The IO Range requirements for the VDS included: 1) Solution for injecting video streams into SIPRNet, 2) Ability to distribute to multiple end user systems at a minimum 1 frame/sec, with seconds of latency, resolution to support projection and over limited bandwidth connections; 3) Ability to record video for playback; and 4) Ability to playback video using a COTS Viewer (Microsoft MediaPlayer).

For IO Range/AC06, the real-time video stream was provided from a single source bridged into the network. The TENA Video Distribution Server published availability of data streams via a VideoStream object and published availability of recorded streams for playback via a VideoStreamServer object. The IO Range VDS supported over thirty clients during the AC06 exercise without a single failure of the TENA Video Distribution Server. The auto-code generated distribution provided by TENA enabled the JMETC developers to greatly reduce the time needed to integrate and test their software. The IO Range brought transformational IO capabilities to the IO community during AC06, providing the warfighter a standing, robust environment for experimentation, testing training, exercises, and operational rehearsal.

JMETC's “Stand Up” event was Integral Fire 07 (IF07), an Air Force Integrated Collaborative Environment (AF-ICE) event completed in August 2007. See Figure 3. IF07 was a distributed test event involving all the military services and JFCOM. JMETC supported the event by providing test infrastructure and technical support. Administered by the Simulation and Analysis Facility (SIMAF) at Wright-Patterson Air Force Base, Ohio, IF07 had three distinct customers: JFCOM's Joint Systems Interoperability Command (JSIC), the DoD Joint Test and Evaluation Methodology (JTEM) Joint Test \& Evaluation (JT\&E) program, and the Warplan-Warfighter 
Forwarder (WWF) initiative, sponsored by the USAF Command and Control Intelligence, Surveillance and Reconnaissance Battlelab. For IF 07, JMETC created a single infrastructure that served these three distinct customers with different requirements who were able to test independently in the same time frame, thereby making multiple use of the same infrastructure.

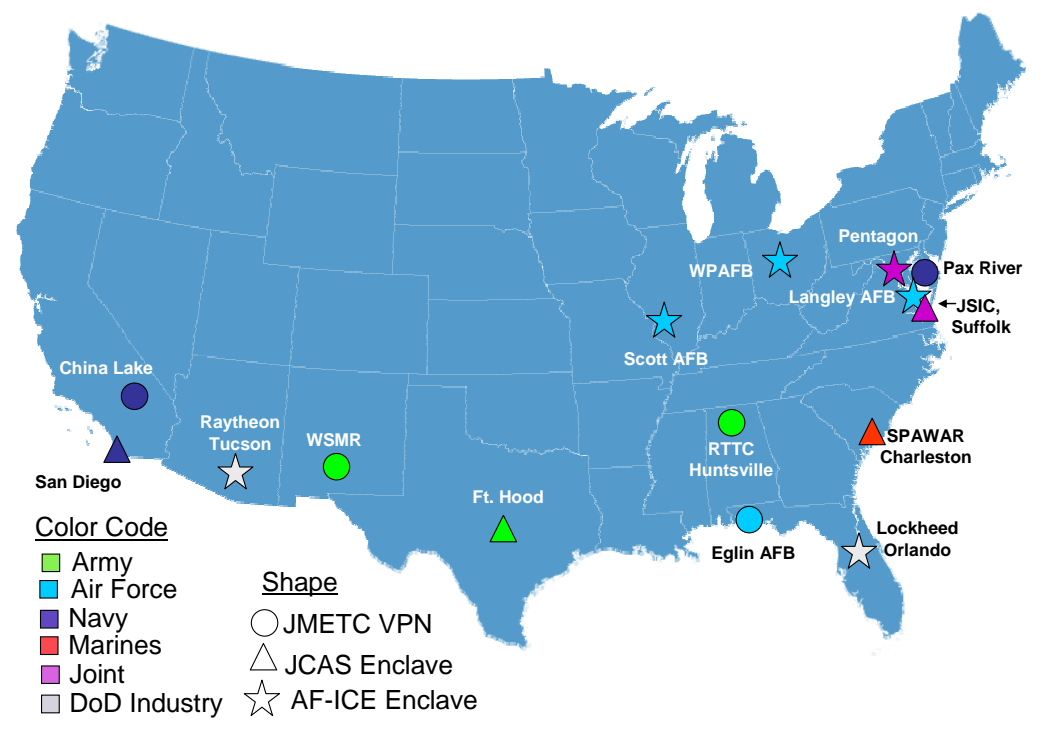

Figure 3. IF07 Locations

Recently, JMETC has directed a large effort to the JC4ISR Interoperability Test and Evaluation Capability (InterTEC) project. InterTEC is being developed and fielded using a spiral development approach. The first spiral focused on developing and fielding an accredited, integrated C4ISR interoperability test capability for the tactical data link protocols of the Joint Data Network (Variable Message Format--VMF, Link 11, and Link 16). The delivery of Spiral 1 in July 2006 constituted the InterTEC Initial Operational Capability (IOC). Spiral 2, recently completed, extends the capability of Spiral 1 to include an integrated test capability for the Joint Planning Network protocols, to include United States Message Text Formatting (USMTF) and Over The Horizon Targeting-Gold (OTH-G). Spiral 3 will focus on intelligence, surveillance, and reconnaissance systems/protocols, as well as supporting the test processes associated with the Net Ready Key Performance Parameter (NR-KPP).

\section{TENA OFFERS INTEROPERABILITY AND RESOURCE REUSE}

Interoperability is the characteristic of an independently developed software element that enables it to work together with other elements toward a common goal. Interoperability focuses on what is common among software elements. Reuse is the ability to use a software element in a context for which it was not originally designed, so reuse focuses on the multiple uses of a single element and requires well-documented interfaces. To achieve interoperability, a common architecture, an ability to meaningfully communicate (including a common language and a common communication mechanism), and a common context (including the environment and time) must be present. To bring the efficiency and economic advantages of interoperability and reuse to the DoD test and training ranges, FI 2010 developed TENA. The FI 2010 program completed the initial interoperability and reuse efforts in early Fiscal Year 2005, and the 
continuing interoperability and reuse refinement of TENA is now managed by the TENA Software Development Activity (TENA SDA).

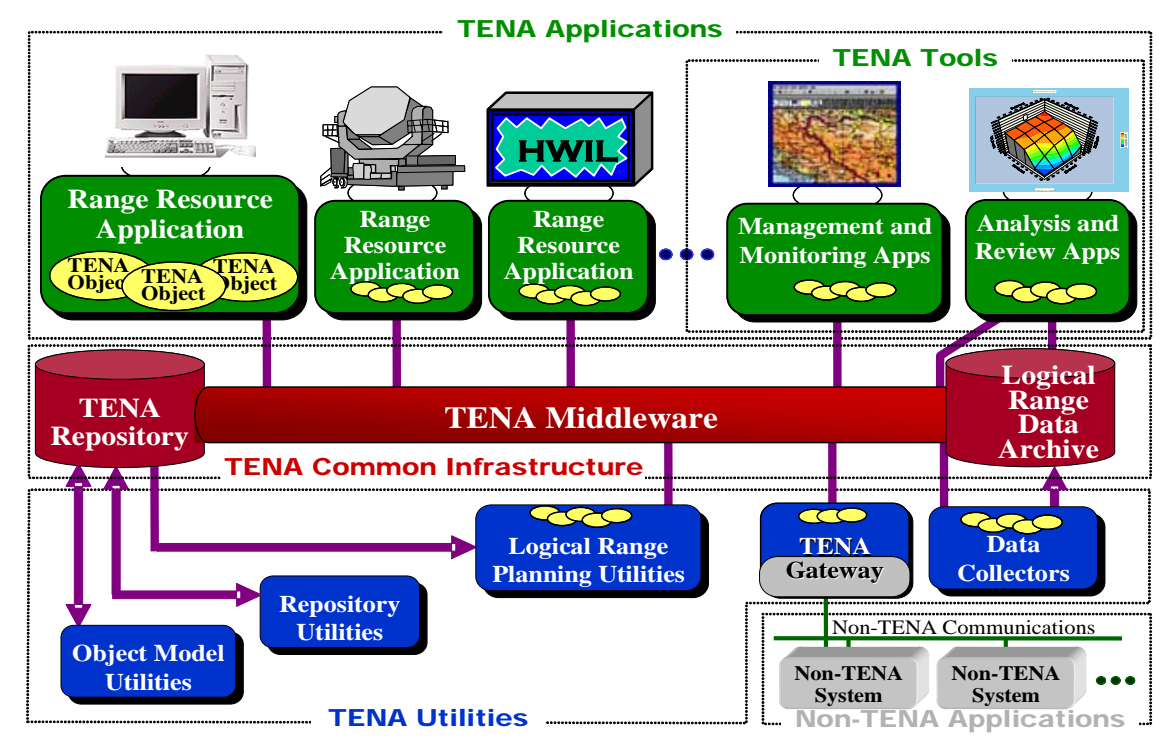

Figure 4. TENA Architecture Overview

The TENA architecture is a technical blueprint for achieving an interoperable, composable set (composibility is defined as the ability to rapidly assemble, initialize, test, and execute a system from members of a pool of reusable, interoperable elements) of geographically distributed range resources-some live, some simulated - that can be rapidly combined to meet new testing and training missions in a realistic manner. Please refer to Figure 4. TENA is made up of several components, including a domain-specific object model that supports information transfer throughout the event lifecycle, common real-time and non-real-time software infrastructures for manipulating objects, as well as standards, protocols, rules, supporting software, and other key components.

The TENA Middleware combines distributed shared memory, anonymous publish-subscribe, and model-driven distributed object-oriented programming paradigms into a single distributed middleware system. This unique combination of high-level programming abstractions yields a powerful middleware system that enables the middleware users to rapidly develop complex yet reliable distributed applications. The TENA Middleware, US Government owned and available for free download at the TENA SDA web site--https://www.tena-sda.com, is currently at Release 5.2.2. Beta versions of Release 6 are available for download.

The TENA object model consists of those object/data definitions, derived from range instrumentation or other sources, that are used in a given execution to meet the immediate needs and requirements of a specific user for a specific range event. The object model is shared by all TENA resource applications in an execution. It may contain elements of the standard TENA object model although it is not required to do so. Each execution is semantically bound together by its object model.

Therefore, defining an object model for a particular execution is the most important task to be performed to integrate the separate range resource applications into a single event. In order to support the formal definition of TENA object models, a standard metamodel has been developed 
to specify the modeling constructs that are supported by TENA. This model is formally specified by the XML Metadata Interchange standard and can be represented by Universal Markup Language. Standards for representing metamodels are being developed under the Object Management Group Model Driven Architecture activities. The TENA Object Model Compiler is based on the formal representation of this metamodel, and TENA user-submitted object models are verified against the metamodel. However, it is important to recognize the difference between the TENA metamodel and a particular TENA object model. The object captures the formal definition of the particular object/data elements that are shared between TENA applications participating in a particular execution while the object model is constrained by the features supported by the metamodel.

A significant benefit for TENA users is auto-code generation. The TENA Middleware is designed to enable the rapid development of distributed applications that exchange data using the publish-subscribe paradigm. While many publish-subscribe systems exist, few possess the highlevel programming abstractions presented by the TENA Middleware. The TENA Middleware provides these high-level abstractions by using auto-code generation to create a complex Common Object Request Broker Architecture (CORBA) application. As such, the TENA Middleware offers programming abstractions not present in CORBA and provides a stronglytype-checked framework interface that is much less error-prone than the existing CORBA API. These higher-level programming abstractions combined with a framework designed to reduce programming errors enable users quickly and correctly to express the concepts of their applications. Re-usable standardized object interfaces and implementations further simplify the application development process.

Through the use of auto code generation, other utilities, and a growing number of common tools, TENA also provides an enhanced capability to accomplish the routine tasks which are performed on the test and training ranges in support of exercises. The steps in many of the tasks are automated, and the information flow is streamlined between tools and the common infrastructure components through the enhanced software interoperability provided by TENA. TENA utilities facilitate the creation of TENA-compliant software and the installing, integrating, and testing of the software at each designated range. This complex task falls to the Logical Range Developer, which, in this phase, performs the detailed activities described in the requirement definitions and event planning, and the event construction, setup, and rehearsal activities of the Logical Range Concept of Operations. While some manual exercise and event setup is required at ranges, TENA tools, as they are developed and become accepted across the range community, will make exercise pre-event management easier.

\section{EMERGING RANGE SYSTEMS AND USE TENA}

TENA's field proven capabilities are being used by other emerging range systems, including the Integrated Network Enhanced Telemetry (iNET) program which provides a solution to capture greater efficiencies in the use of spectrum through revolutionary changes in how flight tests are conducted. TENA is enabling a demonstration prototype to investigate the use of TENA across a constrained environment simulating iNET capabilities. In the process of creating this demonstration, key resource requirements imposed by pairing TENA with iNET for current and anticipated future flight hardware (e.g. size, power, memory, bandwidth) can be measured and/or predicted. Further, creating the demonstration will provide an environment for experimenting with the use of TENA for meeting iNET Metadata requirements. It is expected that performing and documenting this effort will provide guidance to future science and technology topics for 
iNET and TENA. Other systems or events using or planning to use TENA-JMETC include the Technology for Tactical Video (TTV), Integrated Architecture Behavior Model (IABM) in the Single Integrated Air Picture “Joint Combined Hardware-in-the-loop Evaluation” Phase 5 (JCHE5) event, and the Joint Battlespace Dynamic Deconfliction (JBD2) event.

\section{TENA SUPPORT FOR TENA USERS}

TENA SDA has developed a highly utilitarian website that provides a wide range of support for the TENA user, including an easy process to download the middleware which is free. The website also offers a help desk and user forums that will address any problems with the middleware download and implementation. TENA SDA is very aware of the need to inform range managers and train TENA users, and the TENA SDA presents regular training classes that are designed to meet the attendees' needs from an overview of TENA to a technical introduction to TENA to a hands-on, computer lab class for the TENA Middleware.

TENA's continuing evolution in its support of the test and training ranges community is managed by an organization of users and developers. This collection of TENA stakeholders, called the Architecture Management Team, meets every six or eight weeks to be updated on TENA usage, problems, and advancements. The agenda involves briefings and open and wide ranging discussions, and it ensures the users' concerns and inputs are understood, recorded, and made action items, if necessary. Of no less importance, TENA developers and management has had a long and mutually beneficial relationship with the Range Commanders Council.

\section{CONCLUSION}

Although it was a technological and software evolution that was the impetus for TENA's growth in its enabling of range interoperability and resource reuse, the middleware found its needed validation on the DoD test and training ranges. On those ranges, the U.S. Military evaluates the warfighting equipment, personnel, and concepts that are deployed in support of the ongoing missions around the globe. Exercises, experiments, and demonstrations are the stages for the evaluation, but it is the data collection and analysis that determines the war worthiness of the equipment or concept under test. Now paired with JMETC to prove connectivity as well as interoperability and reuse, TENA is being accepted as an important part of the equation. 